\title{
Properties of multielectron bubbles in liquid helium
}

\author{
U. AlbrechT AND P. LEIDERER \\ Fachbereich Phyik, Johannes-Gutenberg-Universität, D-6500 Mainz, Federal Republic of Germany
}

Received Octobcr 13, 1986

\begin{abstract}
We report tharge and velocity measurements on multielectron bubbles in liquid helium. Oscillations and fissioning of bubbles are ohserved.
\end{abstract}

Nous rapportons des mesures de charge et de vitesse sur des bulles multiélectroniques dans l'hélium liquide. Des oscillations et des fissions de ces bulles sont observées.

[Traduit par la revue]

\section{Introduction}

Electrons on the surface of liquid helium are localized in the direction perpendicular to the surface in a potential well consisting of the image potential (plus holding potential) and a potential barrier of $1 \mathrm{eV}$ due to the Pauli exclusion principle (see ref. 1 and references cited therein). Because their motion parallel to the surface is virtually unrestricted, they represent a nearly ideal two-dimensional (2D) Coulomb system. A charged helium surface is stable up to a threshold density of about 2.4 $\times 10^{9}$ electrons $/ \mathrm{cm}^{2}$ (see ref. 2 , ref. 3 , and references cited in ref. 3). Increasing the charge above this threshold density results in an electrohydrodynamic instability of the surface. Dimples with a size of the order of the capillary length appear; they increase in depth until charged macroscopic gas bubbles split off the dimple tip (4). Under the influence of the applied electric holding field $(\geq 3 \mathrm{kV} / \mathrm{cm})$, they are dragged away from the surface and move towards the anode immersed in the liquid, where they discharge and collapse.

These multielectron bubbles are stabilized by the surface tension $\sigma$ and the hydrostatic pressure $p$ in the liquid, counterbalanced by the repulsive Coulomb forces of the electrons. Minimizing the total energy

$$
E=\frac{4}{3} \pi p R^{2}+4 \pi \sigma R^{2}+\frac{Z^{2} c^{2}}{2 \varepsilon R}
$$

of a bubble ( $\varepsilon$ is the dielectric constant of liquid helium) gives the classical "Coulomb radius" of the bubble (this value is accurate for the bubbles considered $(5,6)$ ). For typical charges $Z$ of $10^{5}-10^{7}$ electrons, this yields radii between 10 and $100 \mu \mathrm{m}$. The ele trons in a bubble are confined to a narrow region of only a few angströms width close to the bubble surface, owing to their mutual Coulomb repulsion (5), and thus constitute a spherically symmetric two-dimensional Coulomb system with a considerably higher density $\left(10^{10}\right.$ electrons $/ \mathrm{cm}^{2}$ and more) than in the free-surface case. Thus, the multielectron bubbles might provide information on a regime in the phase diagram of the $2 \mathrm{D}$ electron system that has hardly been accessible to experiments so far.

We report here initial experiments with such bubbles, measuring their charge and concentrating on some dynamical properties like their motion in the liquid helium, oscillations of the bubbles, and their stability.

\section{Experimental details}

All experiments were performed in ${ }^{4} \mathrm{He}$ above the $\lambda$ point. The electron source was a hot tungsten filament mounted inside a small glass tube to localize better the site of bubble production. To charge the surface, we applied a negative potential to

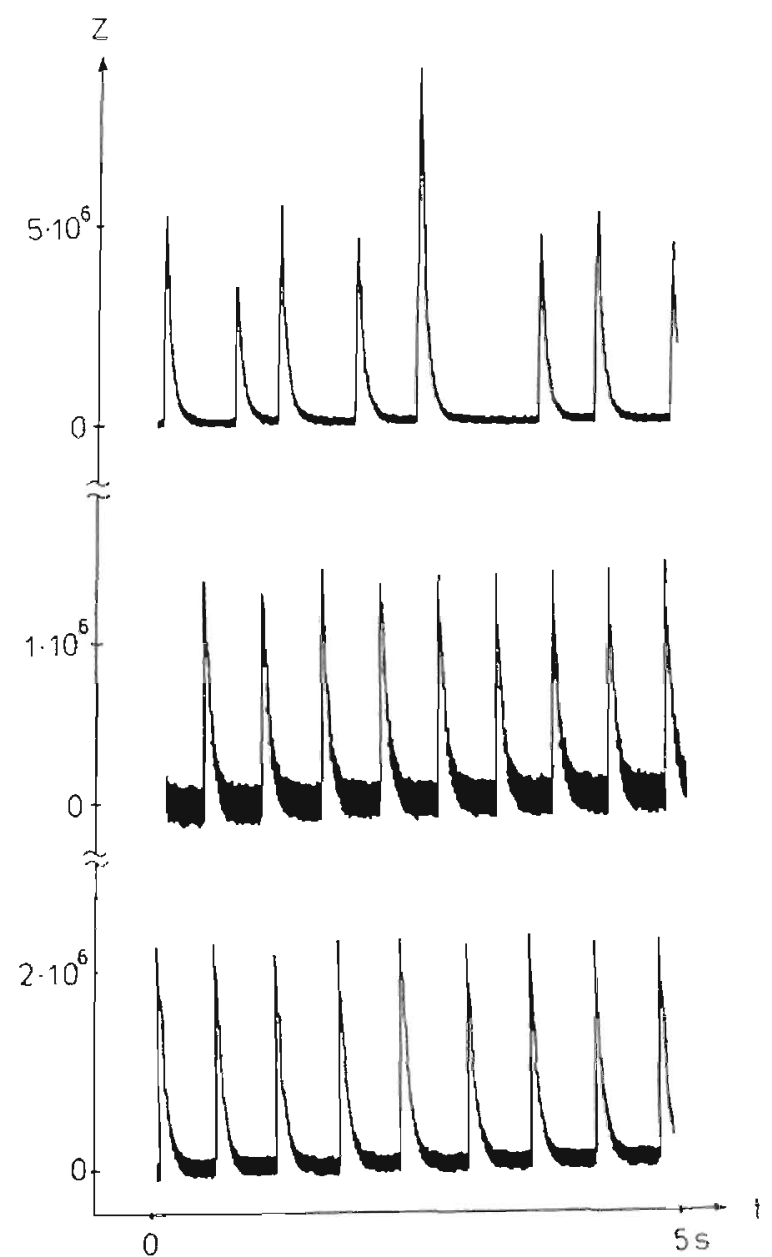

FIG. 1. Displacement current at the anode vs. time duringt continuous bubble production. Each peak corresponds to one bubbli.

the source. By raising the potential to several kilovolts, we could surpass the instability threshold, resulting in continuous bubble production. The bubbles were then dragged to the anode about $2-3 \mathrm{~mm}$ below the liquid-helium surface. We observed them by means of small-angle light scattering using a $\mathrm{He}-\mathrm{Ne}$ laser. Pictures were recorded by a sensitive black and white video camera and stored on tape. The still pictures shown here are photographed from the TV screen using the "single-frame" mode of the video recorder, providing a time resolution of $40 \mathrm{~ms}$.

Bubble charges were measured by recording the displacement current, which is produced when the electron bubble moves towards the anode. Integration of the resulting peaks gave the 


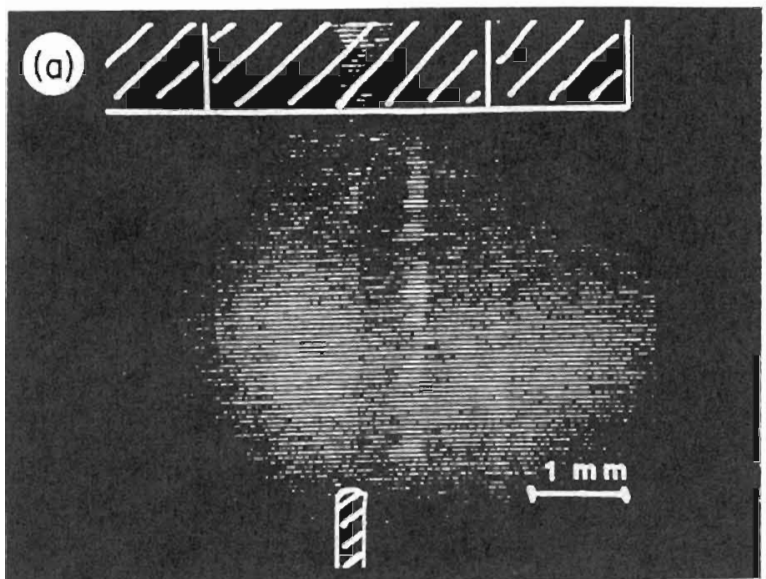

$10 \mathrm{~ms}$

(b)

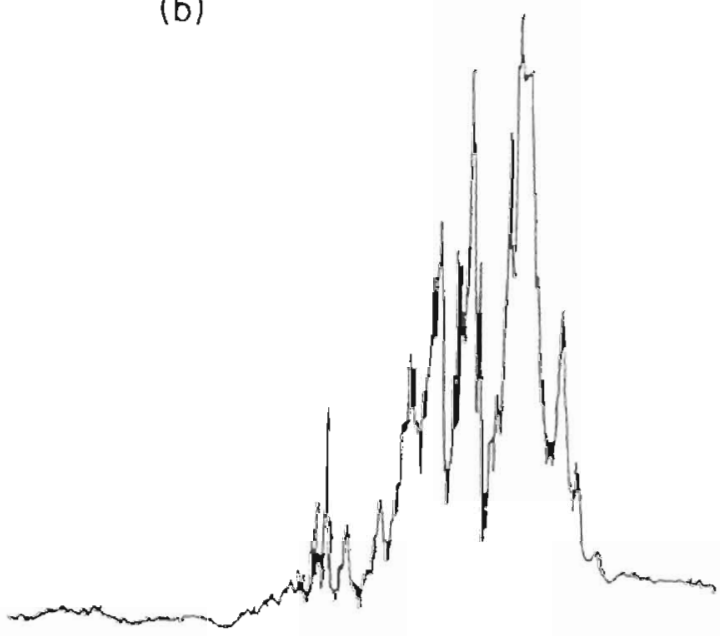

bubble signal

Fig. 2. Oscillating bubble. (a) shows a bubble track, photographed from the TV monitor; $(b)$ is the photomultiplier signal of a similar event. Oscillations of the scattered light intensity with a frequency around $1 \mathrm{kHz}$ are clearly visible.

bubble charge. Typical signals are shown in Fig. 1. We observed charges $Z$ up to about $10^{7}$ electrons. Bubbles with $Z<$ $10^{5}$ were not resolved by our apparatus. The $Z$ values of the bubbles produced depended on the surface tension and surface pressure, the potential gradient at the surface, the electron current from the source, and also the diameter of the glass tube used (about $3 \mathrm{~mm}$ ); the latter determined the boundary conditions for surface oscillations induced by the dimple relaxation after the production of a bubble.

By chopping the laser beam, we also determined the velocity of the bubbles. The bubble tracks then appeared as a sequence of dashes at distances $v / f$ ( $v$ is the bubble velocity and $f$ is the chopping frequency). The bubbles were found to approach a constant velocity quickly after leaving the surface; the velocities ranged from 2 to $15 \mathrm{~cm} / \mathrm{s}$. This implies a time scale of some $10 \mathrm{~ms}$ for the observation of an individual bubble in a visual field of a few millimetres.

Recording bubble charge and velocity simultaneously did not yield any significant correlation. Also, the measured velocities could not be explained by the available data and models on bubble motion in liquids (7). Possible explanations of this lack

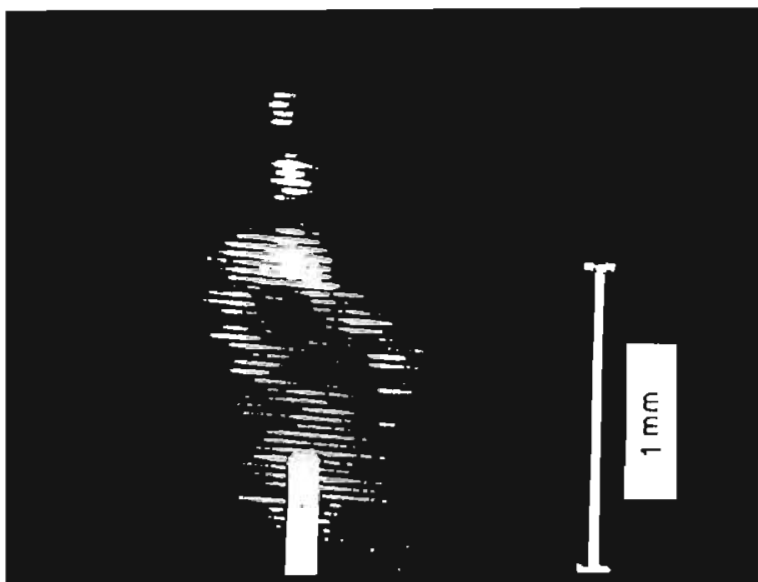

FIG. 3. Track of a fissioning bubble. Here the laser beam was chopped at a frequency $f=370 \mathrm{~Hz}$.

of agreement are the presence of turbulence in the liquid helium and dissipation of energy into oscillations.

\section{Discussion}

We indeed observe such oscillations of the multielectron bubbles. Figure $2 a$ shows the photograph of a bubble track with continuous laser illumination. Obviously the light intensity scattcred from the bubble is not constant along the path but varies noticeably in an oscillatory manner. Figure $2 b$ shows the scattered light intensity recorded with a photomultiplier during a similar event. We interpret these intensity variations as being due to oscillations of the bubbles. This phenomenon is obierved especially well if the bubbles move from an area of high electric field to an area of Jow electric field. The observed main oscillation frequencies are typically around $1 \mathrm{kHz}$, which lies well in the range of the values calculated by Salomaa and co-workers $(5,8,9)$.

Oscillations are related also to the question of dynamical instability of the bubbles. Above a certain deformation amplitude, they are expected to become electrohydrodynamically unstable $(9,10)$. We in fact observe fissioning of bubbles as shown in Fig. 3 (here the laser is chopped). A fast bubble is seen moving downwards and then breaking up into two smaller (lower scattered light intensity) and slower (closer spacing of the dots) bubbles, which move towards the anode tip separately and on curved paths owing to their mutual Coulomb repulsion. Another source of instability is decay by tunneling of single electrons into individual single-electron bubble states (sometimes called "negative ions" (3)), which cannot be observed optically owing to their small size. Because in some cases we could observe individual multielectron bubbles as long as $100 \mathrm{~ms}$ before they left the area of observation, we can state that $\tau>$ $100 \mathrm{~ms}$ for multielectron bubbles under our experimental conditions. At higher electron densities however (achievable by pressurizing the helium), decay by tunneling may become more substantial $(6,10)$.

We hope that more detailed studies on the bubble oscillations and possible trapping in a quadrupole cage (U. Albrecht, P. Leiderer, manuscript in preparation) will extend our knowledge on these interesting systems.

\section{Acknowledgements}

This work was supported by the Deutsche Forschungsgemeinschaft. 
1. F. I. B. Williams. Surf. Sci. 113, 371 (1982); Yu. P. Movarkha and V. B. Shikin. Sov. J. Low Temp. Phys. (Engl. Transl.), 8, 279 (1982).

2. L. P. Gorkov and D. M. Chernikova. JETP Lett. (Engi. Transl.), 18, 68 (1973).

3. P. Leiderer. Physica B + C (Amsterdam), 126, 92 (1985).

4. A. P. Volodin, M. S. KHAIKIN, and V. S. Edflman. JETP Lelt. (Engl. Transl.), 26, 543 (1977); M. S. Khalkin. J. Phy's. Colloq. C6, 1295 (1978).

5. M. M. Salomaa and G. A. Williams. Phys. Scr. T4, 204 (1983).

6. A. A. Artem'ev, A. G. Khrapak, and 1. T. Yakubov. Fiz. Nizk. Temp. (Kiev), 11, 1011 (1985).
7. B. T. Chao. Phys. Fluids, 5, 69 (1962); E. Coester. Z. Angew. Phys. 13, 254 (1961); I. YAron and B. GaL-Or. Int. J. Heat Mass Transfer, 16, 887 (1973).

8. M. M. SalomaA and G. A. Williams. Phys. Rev. Lett. 47, $1730(1981)$

9. S. T. Hannahs, G. A. Williams, and M. M. Salomaa. In Proceedings of the Seventeenth International Conference on Low Temperature Physics. Edited by U. Eckern, A. Schmid, W. Weber, and $\mathrm{H}$. Wühl. North Holland Publishing Company, Amsterdam, The Netherlands. 1984, p. 297.

10. V. B. SHIKIN. JETP Lett. (Engl. Transl.), 27, 39 (1978). 